\title{
Volar Locking Plate Fixation for Intra-Articular Distal Radius Fractures with Volar Lunate Facet Fragments Distal to the Watershed Line
}

\author{
Mitsuhiko Nanno, Norie Kodera, Yuji Tomori and Shinro Takai \\ Department of Orthopedic Surgery, Nippon Medical School, Tokyo, Japan
}

\begin{abstract}
Background: Standard volar plating of distal radius fractures may not adequately fix the volar lunate facet (VLF) fragment, which can result in volar carpal subluxation. We hypothesized that the size of VLF fragments distal to the watershed line might affect reduction loss after distally placed volar locking plate fixation for intra-articular distal radius fracture and examined if the presence of small displaced VLF fragments was a risk factor for reduction loss.
\end{abstract}

Methods: Twenty-seven hands of 27 patients with intra-articular distal radius fractures with VLF fragments distal to the watershed line were treated by using Acu-Loc 2 volar distal radius locking plate fixation.

Results: At final follow-up, the mean Mayo Performance Score was 90.9, and the mean Quick Disabilities of Arm, Shoulder, and Hand score was 13.6. On radiography, 5 patients had a reduction loss of $>2$ $\mathrm{mm}$ in ulnar variance from immediately postoperatively to final follow-up (group 1), while 27 had no reduction loss (group 2). The mean longitudinal, transverse, and anteroposterior lengths and joint surface area of the VLF fragment were significantly smaller in group 1 than in group 2. Three-dimensional computed tomography revealed that the fracture patterns of the radiocarpal and distal radioulnar joints in group 1 were mainly volar-displaced VLF fragments.

Conclusions: By stabilizing fragments, distally placed volar locking plate fixation effectively treated intra-articular distal radius fractures with VLF fragments distal to the watershed line. However, the presence of small displaced VLF fragments may increase the risk of reduction loss in ulnar variance.

(J Nippon Med Sch 2020; 87: 24-31)

Key words: distal radius fracture, volar locking plate, watershed line, distal radioulnar joint, volar lunate facet fragment

\section{Introduction}

Distal radius fracture accounts for $15 \%$ of all fractures and is the most common upper extremity fracture ${ }^{1}$. The standard treatment for distal radius fracture is volar locking plate fixation, which restores anatomy, joint congruity, and stability and enables early rehabilitation and early return to activities of daily living ${ }^{2-9}$. Volar locking plate fixation achieves good clinical results for most distal radius fractures ${ }^{2-9}$.

Recently, many studies have reported that standard volar locking plates designed to be positioned proximal to the watershed line may not be able to adequately stabi- lize and buttress volar lunate facet (VLF) fragments distal to the watershed line in intra-articular distal radius fractures, even though volar locking plate fixation is a wellestablished technique for distal radius fracture ${ }^{2,3,6}$. Standard volar plating of distal radius fractures reportedly leads to inadequate fixation of the VLF fragment, resulting in volar carpal subluxation ${ }^{3}$.

Various distally placed volar locking plates are currently available for treating intra-articular distal radius fractures with fragments distal to the watershed line $e^{4,5,8,9}$. These distally placed plates designed specifically for implantation distal to the watershed line enable direct fixa-

Correspondence to Mitsuhiko Nanno, Department of Orthopedic Surgery, Nippon Medical School Tama Nagayama Hospital,

1-7-1 Nagayama, Tama, Tokyo 206-8512, Japan

E-mail: nanno-mi@nms.ac.jp

https://doi.org/10.1272/jnms.JNMS.2020_87-106

Journal Website (https://www.nms.ac.jp/sh/jnms/) 


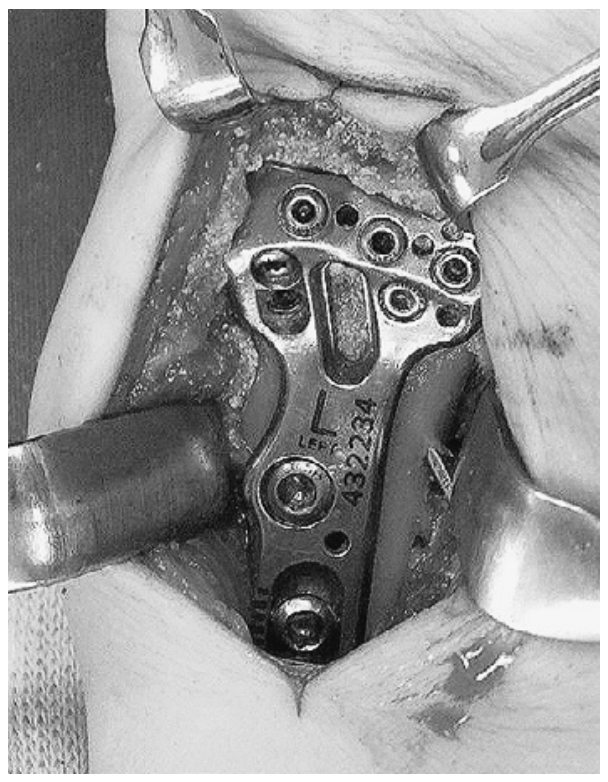

Fig. 1 Fixation with the Acu-Loc 2 distal plate plating system for VLF fragments distal to the watershed line

tion of very distal VLF fragments ${ }^{4,5,8,9}$. Therefore, distally placed plate fixation has been the most commonly used procedure for intra-articular distal radius fractures with VLF fragments distal to the watershed line $e^{4,5,8,9}$. Several studies reported good clinical results and efficacy for distally placed plate fixation ${ }^{4,5,8-11}$. However, some studies noted reduction loss of the VLF fragment or distal radioulnar joint (DRUJ) instability in patients with small VLF fragments, even after treatment with a distally placed volar locking plate ${ }^{4,5,8,9,12}$. One study reported that a small, distally located VLF fragment could not be stabilized with volar distally placed locking plate fixation alone ${ }^{12}$. Even with improvements in distal radius fracture fixation, it remains difficult to stabilize and maintain the VLF fragment in certain fracture patterns ${ }^{2}$.

To date, few studies have noted a correlation between fracture pattern of the radiocarpal joint and DRUJ that involve VLF fragments and reduction loss of VLF fragments in intra-articular distal radius fractures. The VLF fragment involves the sigmoid notch and can affect the stability of the DRUJ ${ }^{14}$. Computed tomography (CT) is useful in accurately assessing the radiocarpal joint and DRUJ surface disruption in relation to VLF fragments ${ }^{14}$.

In the current study, we hypothesized that the size of VLF fragments might affect reduction loss after surgery and investigated if the presence of small displaced VLF fragments was a risk factor for reduction loss. Additionally, we evaluated clinical outcomes of a distally placed volar locking plate for intra-articular distal radius frac- tures with a VLF fragment distal to the watershed line and characterized fracture patterns of cases of intraarticular distal radius fracture with fragments distal to the watershed line in which the DRUJ involving the VLF fragment developed reduction loss. This information will add to our understanding of fracture patterns of the DRUJ in intra-articular distal radius fractures with VLF fragments distal to the watershed line and enable clinicians to select more appropriate treatments with distally placed plate fixation for distal radius fracture, especially for preventing loss of reduction of the VLF fragment.

\section{Materials and Methods}

The present study protocol was approved by our institutional review board and was conducted in accordance with the Declaration of Helsinki. From 2015 through 2018, we recruited 27 consecutive patients with an unstable intra-articular distal radius fracture with VLF fragments distal to the watershed line who were treated with a distally placed volar locking plate. Patients with bilateral distal radius fractures were excluded. All fractures were fixed by using the Acu-Loc 2 distal plate plating system (Acumed, Hillsboro, OR, USA; Fig. 1). This plate is precontoured to the volar rim for placement distal to the watershed line, enabling purchase of the VLF fragment. Acu-Loc 2 distal locking plate fixation is indicated for cases of unstable intra-articular distal radius fracture with VLF fragments distal to the watershed line. Small VLF fragments distal to the watershed line cannot be adequately buttressed with standard volar locking plates. In all cases, the plate was placed distal to the watershed line on the distal radius and was covered by the pronator quadratus and fibrous tissue of the intermediate fibrous $z^{6}{ }^{6}$. In addition, the distally placed plate provided an adequate buttress for the VLF fragment with anchoring fiberwires from the volar joint capsule to the most distal holes of the plate, which addresses a limitation of distally placed volar plate fixation in the management of distal radius fractures involving small VLF fragments. A single surgeon (M.N.) performed all volar locking plate fixation procedures. A short-arm splint applied to immobilize the wrist in a neutral position for 1-3 weeks postoperatively allowed movement of all interphalangeal and metacarpophalangeal joints. Wrist range of motion exercises were started after splint removal. All patients provided written informed consent before examination and treatment.

The clinical results were evaluated at the final followup examination with the Mayo Performance Wrist Score ${ }^{15}$ and the Japanese Society for Surgery of the Hand 


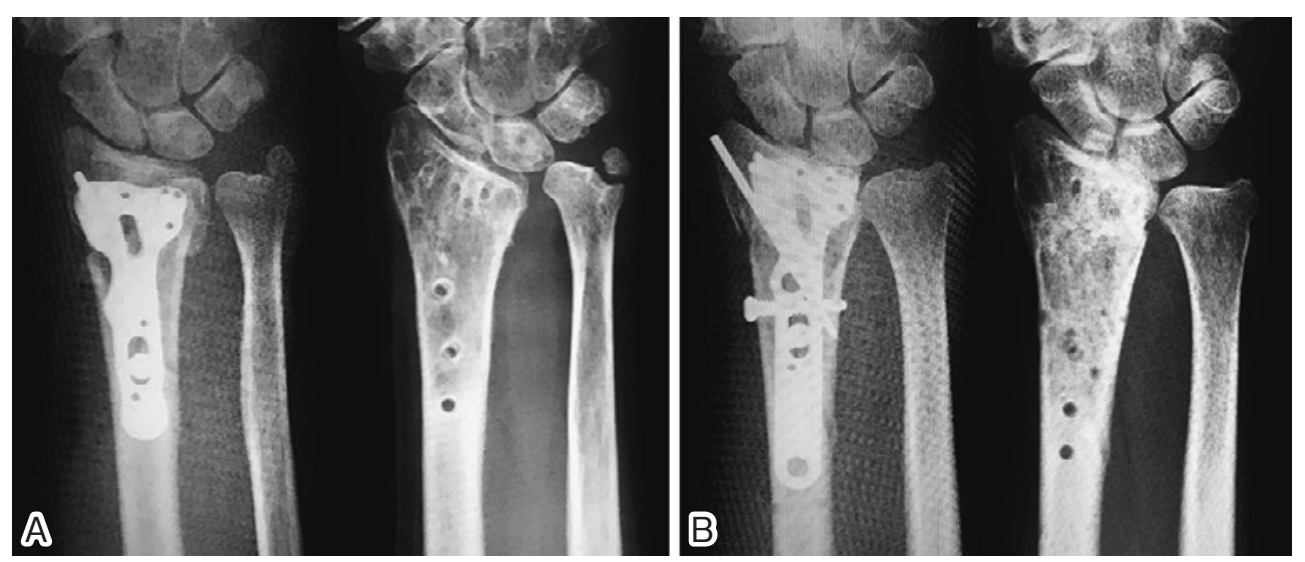

Fig. 2 Anteroposterior radiographs obtained immediately after surgery and at final follow-up in groups 1 and 2

A. Group 1 (reduction loss in ulnar variance), B. Group 2 (no reduction loss in ulnar variance)

Version-Quick Disability of Arm, Shoulder, and Hand questionnaire (Quick DASH-JSSH) ${ }^{16}$ score, a patientoriented questionnaire widely used to assess outcomes of distal radius fracture treatment. The other outcome measures assessed at final follow-up were wrist range of motion, grip strength, and complications.

Three radiographic parameters (volar tilt [VT], radial inclination [RI], and ulnar variance [UV]) were examined on standard anteroposterior and lateral views of the wrist joint obtained in the standardized limb position preoperatively, immediately postoperatively, and at final follow-up. Reduction loss was defined as a change in UV of greater than $2 \mathrm{~mm}$ from immediately postoperatively to final follow-up, in accordance with the method of Ueno et al. ${ }^{7}$. The patients were classified as those with reduction loss (group 1) and those without reduction loss (group 2) (Fig. 2).

All patients underwent preoperative coronal, sagittal, and axial CT imaging, and three-dimensional reconstructive images were created from multislice CT scans (3DCT) (Emotion 6; Siemens, Munich, Germany). The longitudinal and anteroposterior length of the VLF fragment were measured as the distance from the volar ridge of the VLF fragment to the proximal fracture line and the joint surface line, respectively, in the sagittal plane of the CT image, using the software provided with the CT device. Moreover, the transverse length of the VLF fragment was measured as the distance from the palmar edge of the DRUJ to the fracture line in the axial plane of the CT image. The joint surface area of the VLF fragments was measured with free software (image J 1.52a; h ttps://imagej.nih.gov/ij/download.html) in the 3DCT image (Fig. 3).
Fracture patterns of intra-articular distal radius fractures with sigmoid notch involvement were assessed on preoperative CT images in all patients. Rozental and Blazar $^{14}$ classified intra-articular distal radius fractures into 5 fracture patterns on the basis of sigmoid notch involvement and fracture displacement. Type 1a comprises nondisplaced, comminuted fractures with no sigmoid notch involvement. Type $1 \mathrm{~b}$ comprises displaced comminuted fractures with disruption of the radiocarpal joint but without extension into the sigmoid notch. In type 1a and $1 \mathrm{~b}$ fractures, the sigmoid notch fragment, although still congruent with the ulna, is completely separated from the remaining fracture fragments. Type 2 comprises sigmoid notch extension without any fragment displacement (fracture extension into the sigmoid notch without displacement). Type 3a comprises sigmoid notch involvement with rotation and/or translation of the VLF fragment. Type $3 \mathrm{~b}$ comprises sigmoid notch involvement with rotation and/or translation of the volar and dorsal fracture fragments. In the present study, the Rozental classification was modified by adding 2 fracture types (Fig. 4). Fractures that involved the sigmoid notch with rotation and/or translation of the dorsal die punch fragment were defined as type $3 c$, while fractures that involved the sigmoid notch with rotation and/or translation of a free fragment or more than 3 fracture fragments were defined as type 4 (Fig. 4).

All analyses were performed with the software program IBM SPSS Statistics 21.0J (IBM Japan Ltd., Tokyo, Japan). The Student unpaired t-test was used to compare the size of VLF fragments in groups 1 and 2. The association between reduction loss in UV and VLF fragment size was examined. Furthermore, the association between 

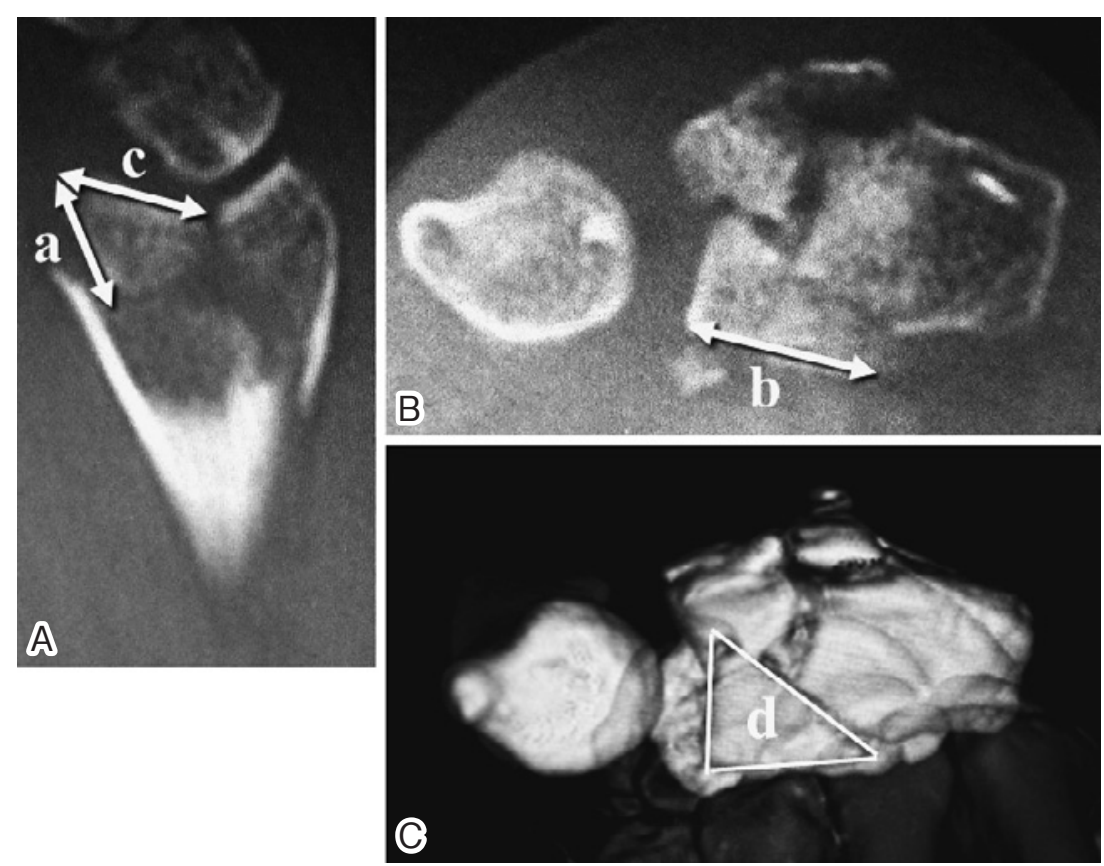

Fig. 3 Computed tomography evaluation of dimensions and area of volar lunate facet fragment

A. Sagittal plane, B. Axial plane, C. Three-dimensional computed tomography

a: longitudinal length, b: lateral length, c: anteroposterior length, d: radiocarpal joint surface area of volar lunate facet fragment

Dorsal side

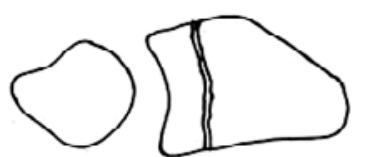

Type 1

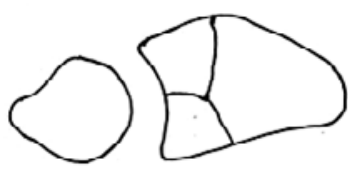

Type 2

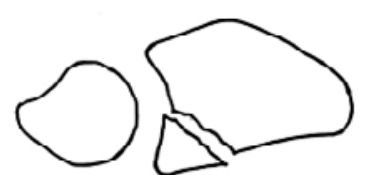

Type 3a

Volar side

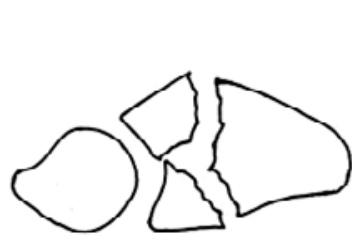

Type $3 b$

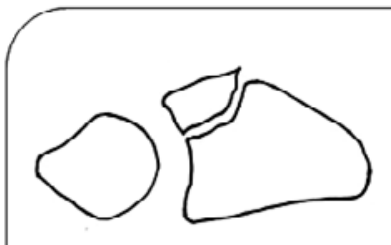

Type 3c

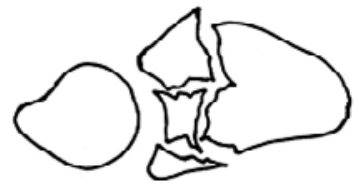

Type 4

\section{Added classification}

Fig. 4 New classification of intra-articular distal radius fractures involving the sigmoid notch on three-dimensional computed tomography

Two additional fracture types were added to the Rozental classification.

Type 1: no sigmoid notch involvement

Type 2: fracture extension into the sigmoid notch, without displacement

Type 3a: sigmoid notch involvement with a displaced volar lunate facet fragment

Type 3b: sigmoid notch involvement with displaced volar and dorsal fracture fragments

Type 3c: sigmoid notch involvement with a displaced dorsal die punch fragment

Type 4: sigmoid notch involvement with a free fragment or more than three fracture fragments 
Table 1 Values for the three radiographic parameters before surgery, immediately after surgery, and at final follow-up

\begin{tabular}{|c|c|c|c|c|}
\hline & \multicolumn{4}{|c|}{ Ragiographic evaluation } \\
\hline & $\begin{array}{l}\text { Unaffected } \\
\text { side hand }\end{array}$ & $\begin{array}{l}\text { Before } \\
\text { surgery }\end{array}$ & $\begin{array}{l}\text { Immediately } \\
\text { after surgery }\end{array}$ & $\begin{array}{c}\text { After } \\
\text { surgery }\end{array}$ \\
\hline & & & \multicolumn{2}{|c|}{ NS } \\
\hline \multirow[t]{2}{*}{ Volar tilt $\left({ }^{\circ}\right)$} & $14.2 \pm 7.6$ & $-10.7 \pm 19.3$ & $8.0 \pm 4.9$ & $8.3 \pm 6.4$ \\
\hline & & & \multicolumn{2}{|c|}{ NS } \\
\hline \multirow[t]{2}{*}{ Radial inclination $\left({ }^{\circ}\right)$} & $25.4 \pm 2.9$ & $11.3 \pm 7.7$ & $23.6 \pm 4.3$ & $23.4 \pm 4.2$ \\
\hline & & & \multicolumn{2}{|c|}{$*$} \\
\hline Ulnar variance (mm) & $1.6 \pm 1.3$ & $2.8 \pm 6.5$ & $1.2 \pm 1.6$ & $2.1 \pm 2.6$ \\
\hline
\end{tabular}

the new classification of the DRUJ fracture and reduction loss in UV was evaluated. A p value of $<0.05$ was considered to indicate statistical significance.

\section{Results}

Distally placed volar locking plate fixation was used to treat 27 patients (27 hands [10 right, 17 left]; 11 men, 16 women; mean age 58.2 years, range $23-79$ years) with unstable intra-articular distal radius fractures with fragments distal to the watershed line. Mean follow-up duration was 15.3 months (range 9-38 months). Fractures were classified in accordance with the AO/ASIF classification system as type C1 $(n=6), C 2(n=2)$, and C3 (n = 19).

Group 1 comprised 22 patients (22 hands [15 left, 7 right]; 9 men, 13 women; mean age 59.7 years, range 2579 years), while group 2 comprised 5 patients ( 5 hands [ 2 left, 3 right]; 2 men, 3 women; mean age 51.6 years, range $23-75$ years). Consequently, $18.5 \%$ (5/27) of cases were evaluated as reduction loss.

There were no significant differences between groups 1 and 2 in age, AO/ASIF classification, or duration of follow-up. Anatomical union was obtained in all patients. At final follow-up, mean Mayo Performance Score was 90.9 (range, 70-100; classified as excellent in 11, good in 15, fair in 1, and poor in 0), mean Quick DASH-JSSH score was 13.6 (range, 6.8-20.5), and mean grip strength was $79.4 \pm 16.8 \%$ of the contralateral side. Mean range of motion in the wrist was $60.0 \pm 8.3^{\circ}$ for volar flexion, 64.7 $\pm 6.8^{\circ}$ for dorsiflexion, $83.5 \pm 12.0^{\circ}$ for internal rotation, and $86.3 \pm 7.3^{\circ}$ for external rotation.

Patients were monitored during the follow-up period for complications, including wound infection, nonunion, tendon rupture, lunate dislocation, subluxation, traumatic osteoarthritis, range of motion limitation, persistent neuropathy, and complex regional pain syndrome. There were no complications in either group, except plate removal. Plate removal was performed in 25 cases (92.6\%); the mean period from internal fixation to implant removal was 7.3 months (range, 5-15 months). No patient developed tendon symptoms.

At final follow-up, mean VT was $8.8^{\circ}$ (range, $-5^{\circ}$ to $23.5^{\circ}$ ), mean RI was $23.4^{\circ}$ (range, $11.3^{\circ}-31.9^{\circ}$ ), and mean UV was $2.1 \mathrm{~mm}$ (range, $0 \mathrm{~mm}-10.5 \mathrm{~mm}$ ) (Table 1). UV was significantly higher at final follow-up than immediately after surgery. VT and RI did not change significantly from immediately after surgery to final follow-up. Mean reduction loss was $0.4 \pm 3.0^{\circ}$ (range, $-4.7^{\circ}$ to $5.1^{\circ}$ ) for $\mathrm{VT}, 0.7 \pm 2.9^{\circ}$ (range, $-2.7^{\circ}$ to $9.7^{\circ}$ ) for RI, and $0.6 \pm$ $0.9 \mathrm{~mm}$ (range, -0.1 to $3.3 \mathrm{~mm}$ ) for UV.

In group 1, VLF fragments had a mean longitudinal length of $6.7 \mathrm{~mm}$, a transverse length of $9.0 \mathrm{~mm}$, an anteroposterior length of $7.6 \mathrm{~mm}$, and a radiocarpal joint surface area of $38.9 \mathrm{~mm}^{2}$. In group 2, VLF fragments had a mean longitudinal length of $8.2 \mathrm{~mm}$, a transverse length of $11.9 \mathrm{~mm}$, an anteroposterior length of $9.4 \mathrm{~mm}$, and a radiocarpal joint surface area of $57.6 \mathrm{~mm}^{2}$. All VLF fragment lengths and VLF area were significantly smaller in group 1 than in group 2 (Table 2).

Fracture pattern was classified as type $2(n=7)$, type 3 a $(n=5)$, type $3 b(n=11)$, and type $4(n=4)$. In group 1 , the classifications were type $3 a(n=1)$, type $3 b(n=1)$, and type $4(\mathrm{n}=3)$.

\section{Discussion}

Several biomechanical studies reported that the centroid 
Table 2 Dimensions and joint surface area of the volar lunate facet fragment on three-dimensional computed tomography images of group 1 (patients with reduction loss) and group 2 (patients with no reduction loss) patients

\begin{tabular}{|c|c|c|}
\hline & \multicolumn{2}{|c|}{ Dimension and area of volar lunate facet fragment } \\
\hline & $\begin{array}{c}\text { Group } 1 \\
\text { (Reduction loss group) }\end{array}$ & $\begin{array}{c}\text { Group } 2 \\
\text { (No reduction loss group) }\end{array}$ \\
\hline \multirow{3}{*}{ Longitudinal length (mm) } & \multicolumn{2}{|c|}{$*$} \\
\hline & $6.7 \pm 2.3$ & $\stackrel{8.2 \pm 2.5}{\longrightarrow}$ \\
\hline & \multicolumn{2}{|c|}{$*$} \\
\hline \multirow[t]{2}{*}{ Transverse length (mm) } & $9.0 \pm 3.6$ & $11.9 \pm 6.1$ \\
\hline & \multicolumn{2}{|c|}{$*$} \\
\hline \multirow[t]{2}{*}{ Anteroposterior length (mm) } & $7.6 \pm 2.7$ & $\stackrel{9.4 \pm 3.6}{\longrightarrow}$ \\
\hline & \multicolumn{2}{|c|}{$*$} \\
\hline $\begin{array}{l}\text { Surface area of } \\
\text { the radiocarpal joint }\left(\mathrm{mm}^{2}\right)\end{array}$ & $38.9 \pm 20.4$ & $\stackrel{57.6 \pm 35.4}{\square}$ \\
\hline
\end{tabular}

of force application is located palmary on the $\operatorname{VLF}^{17}$. The VLF forms an articular portion of the sigmoid notch and serves as the attachment for the short radiolunate ligament $^{13}$. The VLF is considered the keystone of the radiocarpal joint and DRUJ that affects carpal stability ${ }^{11}$. Therefore, failure to fix VLF fragments in distal radius fracture can lead to reduction loss and volar carpal subluxation ${ }^{11}$.

Recently, various distally placed volar locking plates have been used to fix VLF fragments ${ }^{4,5,8,9}$. The indication for distally placed plate fixation is the presence of VLF fragments that are distal to the watershed line and cannot be secured by standard volar plate fixation ${ }^{2-5,7-12}$. However, some studies reported reduction loss of VLF fragments in patients with distal radius fracture and small VLF fragments located very distal to the watershed line, even with the use of a distally placed volar locking plate $^{4,5,8,9,12}$. It is difficult to achieve adequate fixation of small VLF fragments with only a distally placed volar plate, regardless of implant improvements ${ }^{11}$.

Several techniques other than distally placed volar plates have been used to prevent reduction loss and achieve stable fixation of the VLF fragment, including 1) anchoring fiberwires attached to the volar joint capsule to the most distal holes of the plate, to prevent volar displacement of the VLF fragment $\left.{ }^{18}, 2\right)$ supporting the subchondral bone of the VLF fragment via artificial bone grafting in the fracture void, 3) inserting very distal raft screws to support the subchondral bone of the VLF fragment $\left.^{6}, 4\right)$ inserting one or more screws into the VLF fragment, to achieve a distance of $3 \mathrm{~mm}$ or less between the joint surface and screw, 5) additional fixation with Kirschner wires or external fixation, and 6) plate coverage of the VLF fragment of $60 \%$ in the longitudinal direction and $70 \%$ in the transverse direction ${ }^{7}$. In the present study, all these techniques were used to achieve fixation of the VLF fragment.

Some studies in which distal radius fractures with VLF fragments distal to the watershed line were treated with the Acu-Loc 2 distal plate reported good clinical results, with mean reduction losses in UV of 0.45 to $1.19 \mathrm{~mm}^{4,5,8,9}$. The current study achieved a mean reduction loss in UV of $0.7 \mathrm{~mm}$; Acu-Loc 2 distal plate fixation was successfully used to stabilize the VLF fragments with no reduction loss in 5 of 27 patients.

A previous study reported that presence of a VLF fragment with a longitudinal length of less than $15 \mathrm{~mm}$ was a risk factor for volar carpal translation in patients treated with standard volar locking plate fixation ${ }^{2}$. However, few studies have examined the risk factors for reduction loss of the VLF fragment with distally placed volar locking plate fixation. In the present study, the dimensions of the VLF fragment in group 1 (longitudinal length $\leq 7 \mathrm{~mm}$, transverse length $\leq 9 \mathrm{~mm}$, and anteroposterior length $\leq 8 \mathrm{~mm}$ ) were risk factors for reduction loss in UV in patients treated with the distally placed volar locking plate. Thus, the dimensions of the VLF fragment were predictors of reduction loss in UV. It is difficult to stabilize the VLF fragment in fractures with smaller dimensions. The presence of small, displaced VLF fragments, as above, may increase the risk of reduction loss 

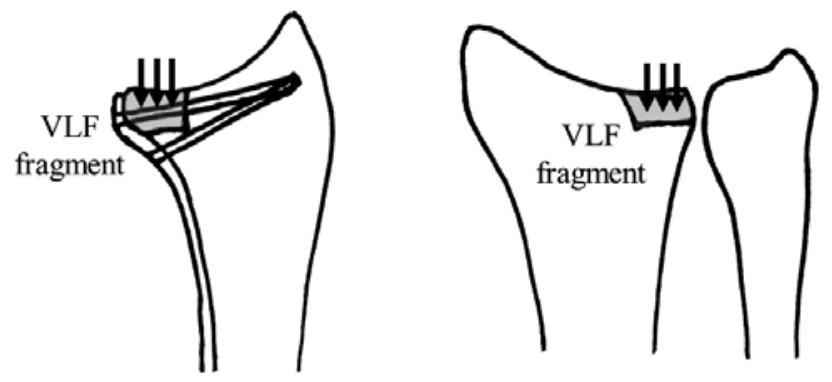

Fig. 5 Displacement and depression of a small volar lunate facet fragment

in UV.

Rozental and Blazar ${ }^{14}$ recommend using CT for preoperative evaluation of comminuted distal radius fractures with sigmoid notch involvement with VLF fragments. Fracture patterns of the sigmoid notch may be important prognostic indicators, and a better understanding of fracture patterns may enable clinicians to secure the VLF fragment and improve clinical outcomes ${ }^{14}$. We modified the Rozental classification to more precisely evaluate fracture patterns in the radiocarpal joint and DRUJ on CT. Consequently, the fracture patterns in most patients who had reduction loss (group 1) were classified as the new type 3, ie, those with a short anteroposterior length and articular surface area of the displaced VLF fragment, or as type 4 , ie, those with a comminuted sigmoid notch and a free VLF fragment.

In the current study, no patient had volar dislocation of the VLF fragment beyond the plate. However, reduction loss in UV occurred in 5 patients with intra-articular distal radius fractures with small VLF fragments distal to the watershed line. Small VLF fragments are reportedly vulnerable to fixation failure, avascular necrosis, and subsequent resorption and often result in volar carpal subluxation ${ }^{6}$. The distally placed plate provided an adequate buttress for the VLF fragment with anchoring fiberwires from the volar joint capsule to the most distal holes of the plate, which addresses a limitation of distally placed volar plate fixation in the management of distal radius fractures involving small VLF fragments; however, very small VLF fragments were still depressed, rotated, or absorbed, resulting in UV reduction loss (Fig. 5). The use of distally placed plate fixation in type 4 fractures may cause reduction loss; thus, it is necessary to further improve the implant or to graft the osteochondral bone to the fractured area in cases with a very small VLF. The present study showed that it is essential to preoperatively evaluate the size of VLF fragments and the fracture pattern of the DRUJ involving the VLF fragments.

\section{Limitations}

The present study had some limitations. First, the sample size was relatively small. A larger sample size might yield more detailed information. Furthermore, this study focused only on a particular type of distal radius fracture with VLF fragments distal to the watershed line and did not include a comparable control group. Additionally, logistic analysis could not be used because of the small sample size. Second, the present data were evaluated retrospectively. Third, all measurements were performed by a single examiner. Therefore, interobserver reliability could not be evaluated. Fourth, no patient underwent arthroscopic or MRI evaluation. Other factors that may cause reduction loss in UV, such as injury to the triangular fibrocartilage complex, could not be evaluated. Finally, duration of follow-up was relatively short. Future studies should attempt long-term evaluation of distally placed volar locking plate fixation for intra-articular distal radius fractures with VLF fragments.

\section{Conclusions}

The current study demonstrated good clinical outcomes for distally placed volar locking plate fixation in intraarticular distal radius fractures with VLF fragments distal to the watershed line. This plating method may be effective in stabilizing the VLF fragment. However, the presence of small displaced VLF fragments may be a risk factor for UV reduction loss in intra-articular distal radius fractures with fragments distal to the watershed line.

Acknowledgements: We thank Kelly Zammit, BVSc, from Edanz Editing (www.edanzediting.com/ac), for editing a draft of this manuscript.

Conflict of Interest: The authors declare no conflicts of interest.

\section{References}

1. Buzzell JE, Weikert DR, Watson JT, Lee DH. Precontoured fixed-angle volar distal radius plates: A comparison of anatomic fit. J Hand Surg. 2008;33A:1144-52.

2. Beck JD, Harness NG, Spencer HT. Volar plate fixation failure for volar shearing distal radius fractures with small lunate facet fragments. J Hand Surg. 2014;39A:6708.

3. Harness NG, Jupiter JB, Orbay JL, Raskin KB, Fernandez DL. Loss of fixation of the volar lunate facet fragment in fractures of the distal part of the radius. J Bone Joint Surg. 2004;86:1900-8.

4. Kura A, Tashima H, Noguchi K, Irie H. [Postoperative clinical results of distal radius fractures involving the distal ulnopalmar fragment distal to the Watershed line]. Orthop \& Traumatol. 2014;63:570-4. Japanese. 
5. Nakamura H, Tanahashi H, Nonomura H, Yokoi T, Nishumura K. [Radiographic outcome and complications of Acu- Loc2 ${ }^{\circledR}$ Volar Distal Radius Plating System for distal radius fractures]. Kossetsu. 2015;37:878-81. Japanese.

6. Orbay JL, Fernandez DL. Volar fixation for dorsally displaced fractures of the distal radius: a preliminary report. J Hand Surg. 2002;27A:205-15.

7. Ueno Y, Kawasaki K, Okano I, Kuroda T, Inagaki K. [Correlation between support for volar lunate facet fragments and loss of reduction in the C3 type distal radius fractures]. J Jpn Soc Surg Hand. 2018;34:724-9. Japanese.

8. Ugawa R, Morishita T, Kadota H, Okada Y, Yokoyama Y, Watanabe M. [Clinical outcome of the treatment of distal radius fractures with Acu-Loc 2 plate: comparison with traditional Acu-Loc plate]. Kossetsu. 2015;37:882-5. Japanese.

9. Yoshimura T. [Experience of surgical treatments for distal radius fracture with Aculoc2 ${ }^{\circledR}$ produced by ACUMED company]. Cent Jpn J Orthop Traumat. 2014;57:1219-20. Japanese.

10. Lee JK, Lee SH, Sim WS, Kim TH, Baek E, Han SH. Juxta-articular plate fixation in distal radius intra-articular fractures with accompanying volar free fragments beyond the watershed line. Clin Orthop Surg. 2018;10:135-41.

11. Kitay A, Mudgal C. Volar carpal subluxation following lunate facet fracture. J Hand Surg Am. 2014;39:2335-41.

12. Moore AM, Dennison DG. Distal radius fractures and the volar lunate facet fragment: Kirschner wire fixation in addition to volar-locked plating. Hand. 2014;9:230-6.

13. Berger RA, Landsmeer JM. The palmar radiocarpal ligaments: a study of adult and fetal human wrist joints. J Hand Surg. 1990;15A:847-54.

14. Rozental TD, Blazar PE. Functional outcome and complications after volar plating for dorsally displaced, unstable fractures of the distal radius. J Hand Surg. 2006;31:35965.

15. Cooney WP, Linscheid RL, Dobyns JH. Triangular fibrocartilage tears. J Hand Surg Am. 1994;19:143-54.

16. Imaeda $\mathrm{T}$, Toh $\mathrm{S}$, Wada $\mathrm{T}$, et al. Validation of the Japanese Society for Surgery of the Hand Version of the Quick Disability of the Arm, Shoulder, and Hand (QuickDASHJSSH) questionnaire. J Orthop Sci. 2006;11:248-53.

17. Genda E, Horii E. Theoretical stress analysis in wrist joint-neutral position and functional position. J Hand Surg. 2000;25B:292-5.

18. Watanabe K. [Treatment of distal radius fracture with DVR anatomic hook plate]. J MIOS. 2015;75:27-34. Japanese.

(Received, June 10, 2019)

(Accepted, September 4, 2019)

(J-STAGE Advance Publication, October 15, 2019) 